\title{
Short-Term Distribution Kinetics of Intratracheally Administered Exogenous Lung Surfactant
}

\author{
JONATHAN M. DAVIS, GERALD A. RUSS, LEON METLAY, BRYON DICKERSON, AND
} BENNETT S. GREENSPAN

\begin{abstract}
Departments of Pediatrics (Neonatology), Radiology, Pathology and Laboratory Medicine, and the Specialized Center of Research in Respiratory Disorders of Newborns, University of Rochester School of Medicine and Dentistry, Rochester, New York 14642
\end{abstract}

\begin{abstract}
The short-term distribution kinetics of exogenous surfactant distribution after intratracheal instillation was investigated in surfactant-deficient neonatal piglets during assisted conventional mechanical ventilation and by high-frequency jet ventilation using exogenous calf lung surfactant extract (CLSE) labeled with ${ }^{99 m}$ Tc. Surfactant deficiency was induced by repeated bronchoalveolar lavage in piglets $(1.2 \pm 0.4 \mathrm{~kg}, 1.4 \pm 0.7 \mathrm{~d}$ of age $)$, and the short-term distribution kinetics of instilled, labeled CLSE were followed by gamma radioscintigraphy. Animals ventilated by either conventional mechanical ventilation or high-frequency jet ventilation showed similar improvement in arterial/alveolar oxygen ratios after surfactant replacement therapy $(0.47 \pm \mathbf{0 . 0 3}$ prelavage, $0.09 \pm 0.01$ postlavage, $0.36 \pm 0.06$ postsurfactant). This correlated directly with dynamic radioscintigraphic results showing that instilled CLSE began to distribute to the lungs within $5 \mathrm{~s}$, and was present in substantial amounts in standardized symmetrical lung fields (central, right, and left; upper and lower) within $20 \mathrm{~s}$ of tracheal instillation. Subsequent measurements over $30 \mathrm{~min}$ showed continued presence of radiolabeled CLSE in all five areas of the lung, with no significant difference between conventional mechanical ventilation and high-frequency jet ventilation animals. Static (5-min) analyses at the end of this period showed that surfactant had distributed relatively symmetrically with $30 \%$ of the CLSE located in central regions, $40 \%$ in the upper lobes, and $30 \%$ in the lower lobes. In contrast, piglets receiving ${ }^{99 m} \mathrm{Tc}$ in saline showed nonuniform distribution with multiple filling defects noted throughout the lungs. The rapid kinetics and ventilation independence of CLSE distribution suggest that surfactant spreading phenomena after tracheal instillation may faciltiate the delivery of exogenous surfactant into aerated lungs in therapeutic applications. (Pediatr Res 31: 445-450, 1992)
\end{abstract}

\section{Abbreviations}

CMV, conventional mechanical ventilation

HFJV, high-frequency jet ventilation

CLSE, calf lung surfactant extract

RDS, respiratory distress syndrome

BAL, bronchoalveolar lavage

TCA, trichloroacetic acid

Received March 27, 1991; accepted December 19, 1991

Correspondence: Jonathan M. Davis, M.D., Division of Newborn Medicine, Winthrop University Hospital, 259 First Street, Mineola, NY 11501.

Supported by a Specialized Center of Research Grant (SCOR) HL-36543 and PHS Grant 2-507-RR05403.
RDS is a major cause of morbidity and mortality in premature infants who are born before full maturation of the pulmonary surfactant system (1). Because a deficiency in endogenous surfactant is a major factor contributing to the development of RDS, a good deal of research has been directed toward the development of effective exogenous surfactant replacement therapy $(2,3)$. A number of clinical trials have now shown that exogenous surfactant preparations can be delivered by intratracheal instillation to premature infants with RDS, resulting in improved gas exchange, pulmonary mechanics, and clinical status (4-8). The presumed mechanisms of action of instilled surfactant in infants are at the alveolar level, with a lowering in surface tension forces, decreased atelectasis, and better matching of ventilation to perfusion $(2,3)$. However, little is known quantitatively about the kinetics of the process by which instilled surfactant distributes from the trachea into the lung. Little information is also available on how the dynamics of this distribution process might be affected by different modes of assisted ventilation.

In general, the quantitative assessment of exogenous surfactant distribution in lungs is a complex problem and requires the ability to analyze in detail the concentration of surfactant as a function of position within the airways and alveoli. However, if one is interested primarily in examining the time scale of initial movement of surfactant into the lungs after tracheal instillation, then dynamic, qualitative assessments would be of significant importance.

In the present article, we examine the short-term distribution kinetics of an exogenous CLSE after intratracheal instillation into newborn piglets made surfactant deficient by repeated lung lavage. A stannous reagent reduction technique is used to directly label molecular constituents in CLSE with ${ }^{99 \mathrm{~m}} \mathrm{Tc}$, and the dynamics of the surfactant distribution process are then measured by gamma scintigraphy. In addition, two types of assisted ventilation that give rise to different respiratory flow characteristics are studied in terms of their effects on surfactant distribution: CMV and HFJV. The first of these ventilation modes is routinely used clinically in the treatment of infants with RDS and involves gas exchange by bulk flow with tidal volumes that substantially exceed pulmonary dead space during each breath. The latter technique of HFJV has been used successfully to treat infants with severe respiratory failure who are unresponsive to conventional ventilation (9). It generates tidal volumes that are less than dead space and relies on gas transport mechanisms (i.e. turbulent flow) related to the high frequency of operation (10). However, these two different ventilation techniques were not found to generate significant differences in CLSE distribution kinetics, suggesting that mechanisms other than bulk mixing and transport contribute to initial surfactant movement. 


\section{MATERIALS AND METHODS}

Exogenous surfactant (CLSE). Detailed descriptions of the CLSE preparation used here have been reported previously $(2$, $3,5,11)$. Briefly, this material was prepared by organic solvent (chloroform-methanol) extraction of bronchoalveolar-lavage surfactant from calves. CLSE contains $98-99 \%$ lipid, with a component distribution identical to that of natural surfactant. In addition, the extract has between 1 and $2 \%$ protein by weight, which is largely hydrophobic SP-B and SP-C lung surfactant apoprotein necessary for rapid adsorption $(2,3)$. CLSE has been used extensively in clinical trials of surfactant replacement therapy in infants with $\operatorname{RDS}(4,7,8)$ and was used as a representative material for the exogenous surfactant distribution studies here.

Radionuclide labeling of exogenous surfactant. ${ }^{99 \mathrm{~m}} \mathrm{Tc}$ labeling of CLSE was performed in the presence of stannous reducing agent. Initially, $1 \mathrm{~g}$ of stannous chloride $\left(\mathrm{SnCl}_{2} \cdot \mathrm{H}_{2} \mathrm{O}\right)$ was dissolved in $10 \mathrm{~mL}$ of concentrated hydrochloric acid, and the solution was filtered through a $0.22 \mu \mathrm{m}$ filter. A $1-\mathrm{mL}$ aliquot of filtrate was diluted 100 -fold with deionized distilled water and the $\mathrm{pH}$ was adjusted to 7.1-7.5, with a final concentration of stannous ion of $572 \mu \mathrm{g} / \mathrm{mL}$. Two $\mathrm{mL}$ of this stannous reducing reagent were then combined with $0.5 \mathrm{~mL}$ of ${ }^{99 \mathrm{~m}} \mathrm{Tc}(10 \mathrm{mCi})$ and $2.5 \mathrm{~mL}$ of CLSE in $0.15 \mathrm{M} \mathrm{NaCl}(60 \mathrm{mg} / \mathrm{mL})$. The labeled CLSE (now $30 \mathrm{mg} / \mathrm{mL}$ ) was then stirred and incubated at room temperature for at least 3 min before instillation into animals. After labeling, the surface activity of CLSE was analyzed with a pulsating bubble apparatus (2) to verify that its ability to lower surface tension had not been affected. Both labeled and unlabeled CLSE reached minimum surface tensions $<1$ dyne $/ \mathrm{cm}$ at $37^{\circ} \mathrm{C}$ on the bubble at a phospholipid concentration of $1 \mu \mathrm{mol} / \mathrm{mL}$.

For kinetic studies, it was important to verify that the ${ }^{99 \mathrm{~m}} \mathrm{Tc}$ label was bound to surfactant components and not easily separated during distribution. For this purpose, it was not necessary to define the precise molecular location of ${ }^{99 \mathrm{~m}} \mathrm{Tc}$, although one likely target for labeling is exposed $\mathrm{COOH}$ groups on surfactant apoproteins. Five complementary assays were used here to characterize ${ }^{99 \mathrm{~m}}$ Tc-labeled CLSE. The first of these was thin-layer chromatography, which was performed using instant thin-layer chromatography Fiberglas strips layered with silica gel (ITLCSG, Gelman Instrument Co., Ann Arbor, MI). Ten to $20 \mu \mathrm{L}$ of labeled CLSE were applied to the dry strip $1 \mathrm{~cm}$ from the bottom. The strip was allowed to run and was developed with acetone. In this system, unreacted pertechnetate migrates near the solvent front, whereas reduced ${ }^{99 \mathrm{~m}} \mathrm{Tc}$ produced in the absence of a binding ligand does not migrate appreciably from the origin (12).

Assessments of ${ }^{99 \mathrm{~m}} \mathrm{Tc}$ binding were also performed by dialysis, TCA precipitation, and cellulose acetate electrophoresis. Dialysis of CLSE dispersions after labeling was carried out for $2 \mathrm{~h}$ at room temperature in a microdialysis tube (molecular weight cutoff approximately 10000$)$ without a change of dialysate $(0.9$ $\mathrm{N} \mathrm{NaCl}, 1: 10000)$. Measurements of dialyzed radioactivity then defined the percentage of label that was either free, loosely bound, or bound to small dialyzable molecules in CLSE. For TCA precipitation, equal parts of labeled surfactant and $10 \%$ TCA were mixed, giving rise to a protein-containing precipitate that was subsequently centrifuged $(1300 \times g)$ and washed. The radioactivity retained in the washed pellet determined bound label. Cellulose acetate electrophoresis measurements were performed by spotting a few nanoliters of labeled surfactant on a saturated cellulose acetate membrane, which was developed for $30 \mathrm{~min}$ in a barbital buffer (pH 8.0) at a constant voltage of $250 \mathrm{mV}$. The membrane was stained with Ponceau red stain and washed several times in $5 \%$ acetic acid to disclose the protein bands, and the ${ }^{99 \mathrm{~m}} \mathrm{Tc}$ activity was determined. Finally, labeled surfactant was stringently exposed to organic solvent extraction to confirm that the label was tightly bound (13). As shown later, all these measurements confirmed that CLSE was directly labeled with ${ }^{99 \mathrm{~m}}$ Tc.

Physiologic studies. Seventeen newborn piglets weighing $1.2 \pm$
$0.4 \mathrm{~kg}$ (mean $\pm \mathrm{SEM}$ ) and $1.4 \pm 0.7 \mathrm{~d}$ of age were studied. At the start of each experiment, piglets were anesthetized with intraperitoneal pentobarbital $(25 \mathrm{mg} / \mathrm{kg})$, placed under a radiant warmer, and intubated (with either a conventional or highfrequency "hi-lo" endotracheal tube), and an umbilical arterial line was inserted. Mechanical ventilation was begun in all animals with a Bournes (Riverside, CA) BP 200 Ventilator with inspiratory time $0.5 \mathrm{~s}, 100 \%$ inspired oxygen, and a flow rate of $8 \mathrm{~L} / \mathrm{min}$. Positive end expiratory pressure was maintained at 3$4 \mathrm{~cm} \mathrm{H}_{2} \mathrm{O}$ in all animals, but peak inspiratory pressure and ventilator rate were varied as necessary to keep arterial $\mathrm{CO}_{2}$ pressure at $5.2-5.8 \mathrm{kPa}(40-45 \mathrm{mmHg})$. Proximal airway pressure was measured with a Novametrix (Wallingford, CT) pressure monitor, and arterial blood gases were measured by a Corning (Medfield, MA) 168 blood gas analyzer. Five percent dextrose with $0.25 \mathrm{~N}$ saline was administered i.v. at $80 \mathrm{~mL} / \mathrm{kg} / \mathrm{d}$, and additional pentobarbital was administered as needed to maintain sedation.

Arterial blood gases were obtained and pulmonary function testing was performed on all experimental animals (supine) at time zero using a computerized technique (MAS, Hatfield, PA) $(14,15)$. Assessments for pulmonary mechanics required a 1min measurement period, during which flow rates were measured with a pneumotachometer (Fleisch 00; OEM Medical Inc., Richmond, VA) inserted between the endotracheal tube and ventilator, and an esophageal balloon (in the lower third of the esophagus) was used to estimate pleural pressure. Transpulmonary pressure was measured with a differential pressure transducer (Validyne Engineering Corp., Northridge, CA), and was expressed as airway minus esophageal pressure. Flow and pressure signals were continuously relayed to a computer and digitized at $75 \mathrm{~Hz}$. Flow was then numerically integrated to give tidal volume, and minute ventilation was calculated. In addition, total dynamic lung compliance (corrected to weight, not lung volume) and pulmonary resistance were determined from flow and pressure signals by two-factor least mean square analysis (15). Computer analysis permitted graphic evaluation and screening of individual breaths by simultaneous display of scalar tracings, flow-volume, and pressure-volume relationships. Only mechanical ventilator breaths that were complete and nondistorted were selected for final analysis, with a mean value reported for a minimum of 10 breaths.

After pulmonary function testing, surfactant deficiency was induced in the neonatal piglets by repeated BAL with warm saline (16). BAL was performed with $50-\mathrm{mL}$ aliquots of saline at 5 -min intervals until the arterial $\mathrm{O}_{2}$ pressure was less than 12.9 $\mathrm{kPa}(100 \mathrm{mmHg})$ and total dynamic lung compliance had decreased by more than $50 \%$. In general, this required five to six repeated lavages for the piglets studied here, with $>60 \%$ lavage volume recovery for all animals. In a subgroup of animals $(n=$ $3)$, BAL was centrifuged $(400 \times g, 10 \mathrm{~min})$ to remove cells and was analyzed on an aliquot-by-aliquot basis for phospholipid (17) to quantitate surfactant washout: lavage $1,1.1 \pm 0.0 \mu \mathrm{mol}$ phospholipid/mL (mean \pm SEM); lavage 2, $0.8 \pm 0.1$; lavage 3 , $0.6 \pm 0.1$; lavage $4,0.5 \pm 0.1$; lavage $5,0.4 \pm 0.1$; and lavage 6 , $0.2 \pm 0.1$.

After BAL, pulmonary function testing was again performed on all animals. Animals were then subdivided into four groups as follows: 1) Three piglets were killed and lung sections were examined microscopically to demonstrate compromised inflation patterns; 2) six piglets were given $100 \mathrm{mg} / \mathrm{kg}$ of labeled CLSE as a single intratracheal bolus followed by distributional assessments over $30 \mathrm{~min}$ while being ventilated with CMV; 3) five piglets were given an identical dose of labeled CLSE and studied over 30 min while receiving HFJV (Bunnell Inc., Salt Lake City, UT); and 4) three piglets received ${ }^{99 \mathrm{~m}} \mathrm{Tc}$ in saline and then $30 \mathrm{~min}$ of CMV $(n=2)$ or $\operatorname{HFJV}(n=1)$.

All experimental animals were initially conventionally ventilated with similar proximal mean airway pressures $(6.0 \pm 0.5 \mathrm{~cm}$ $\mathrm{H}_{2} \mathrm{O}, \mathrm{CMV} ; 5.4 \pm 0.3 \mathrm{~cm} \mathrm{H} \mathrm{H}_{2} \mathrm{O}$, HFJV; $\left.p=\mathrm{NS}\right)$. When animals 
in the HFJV group were initially conventionally ventilated, both proximal (mouth) and distal (tip of the endotracheal tube) mean airway pressures were measured. After labeled CLSE or saline was administered, animals were placed on HFJV with ventilator settings typical for clinical applications in neonates, including a rate of 420 breaths $/ \mathrm{min}$, inspiratory time of $0.02 \mathrm{~s}$, and a positive end expiratory pressure of $3 \mathrm{~cm} \mathrm{H}_{2} \mathrm{O}$, which provided adequate gas exchange with minimal air trapping. Inspiratory pressure was varied to keep distal mean airway pressure constant during ventilation with CMV and subsequent HFJV. This ensured that mean airway pressure was comparable in both ventilation groups. A background sigh rate of 4 breaths/min was provided by the conventional ventilator during $\mathrm{HFJV}$, with the inspiratory pressure maintained $2-3 \mathrm{~cm} \mathrm{H}_{2} \mathrm{O}$ below that of the jet ventilator as is commonly done clinically.

During the 30 -min experimental ventilation period, dynamic surfactant distribution in CMV and HFJV animals was continuously imaged using a large field of view gamma camera (Searle, Arlington Heights, IL). Imaging was performed in a dynamic mode using a $128 \times 128$ matrix. Data were collected in 5-s frames for the first minutes after surfactant administration and then every $30 \mathrm{~s}$ for the remainder of the 30 -min study. Data was stored in an Informatek (Paris, France) computer that had been fitted with Sopha Software (Sopha Development Software, Gaithersburg, MD). Five separate regions of interest were defined (central, right upper, right lower, left lower, and left upper) over the lungs, and regional activity curves for labeled CLSE were constructed as a function of time. At the conclusion of the dynamic study, a 5-min static image for counts in each lung region was also acquired and stored, along with repeat blood gases and pulmonary function studies. The continuous 5-min static counting period provides a more accurate assessment of ultimate surfactant distribution than the shorter counting periods (5-30 sec) used during dynamic imaging. Lung sections were then obtained for light microscopy to examine overall inflation patterns in surfactant-treated animals compared with lavaged animals not receiving surfactant.

Microscopic studies. Qualitative evaluations of overall inflation patterns were performed in the 17 study animals and in six additional piglets used as unventilated controls. When the animals were killed $(150 \mathrm{mg} / \mathrm{kg}$ pentobarbital), the chest was opened and a catheter was inserted into the right ventricle and secured in the main pulmonary artery (18). The left atrium was opened to allow for escape of infused fluids. While the lungs were maintained at constant airway inflating pressure of $20 \mathrm{~cm} \mathrm{H}_{2} \mathrm{O}$, $60 \mathrm{~mL}$ of normal saline were slowly infused to wash out blood from the pulmonary circulation. Then, $100 \mathrm{~mL}$ of phosphatebuffered paraformaldehyde/glutaraldehyde were perfused slowly through the vascular system over $15 \mathrm{~min}$. After fixation, the lungs were removed, and random tissue blocks from each lobe were processed for light microscopy. Light microscopic specimens were embedded in paraplast, sectioned, and stained with hematoxylin and eosin. Sections were examined for abnormal inflation patterns in a blinded fashion. Multiple fields were examined and graded according to the degree of atelectasis (i.e. mild, moderate, or severe). The pathologist was blinded to specific treatments of individual piglets.

Statistical analysis. Physiologic changes occurring pre- and postlavage and postsurfactant replacement were analyzed by oneway analysis of variance, as were radioactive count differences in various lung fields during surfactant distribution. Differences between specific variables in the CMV and HFJV groups were analyzed by unpaired $t$ test.

\section{RESULTS}

The extent of ${ }^{99 \mathrm{~m}} \mathrm{Tc}$ binding to CLSE during the labeling process is assessed in Table 1, and the first four methods studied indicated binding of $>90 \%$ of original radiolabel. Instant thinlayer chromatography demonstrates the highest degree of ${ }^{99 \mathrm{~m}} \mathrm{Tc}$
Table 1. Determination of extent of surfactant label*

\begin{tabular}{lc}
\hline \multicolumn{1}{c}{ Assay used } & \% label \\
\hline ITLC & $99.5 \pm 0.3(n=5)$ \\
Dialysis & $93.7 \pm 1.5(n=4)$ \\
TCA precipitation & $93.8 \pm 0.6(n=5)$ \\
Cellulose acetate electrophoresis & $91.1 \pm 1.8(n=4)$ \\
Organic solvent extraction & $59.0 \pm 2.0(n=2)$ \\
\hline
\end{tabular}

* Results are expressed as the mean percent $( \pm$ SEM $)$ of ${ }^{99 \mathrm{~m}} \mathrm{Tc}$ that was directly associated with surfactant. $n$ indicates number of samples of labeled CLSE studied. ITLC, instant thin-layer chromatography.

Table 2. Physiologic variables in piglets*

\begin{tabular}{lccc}
\hline & $\begin{array}{c}\text { Prelavage } \\
(n=17)\end{array}$ & $\begin{array}{c}\text { Postlavage } \\
(n=17)\end{array}$ & $\begin{array}{c}\text { Post- } \\
\text { surfactant } \\
(n=11)\end{array}$ \\
\hline $\begin{array}{c}\text { Arterial/alveolar oxy- } \\
\text { gen ratio }\end{array}$ & $0.47 \pm 0.03$ & $0.09 \pm 0.01$ & $0.37 \pm 0.05$ \\
$\begin{array}{c}\text { Tidal volume (mL/ } \\
\text { kg) }\end{array}$ & $14.7 \pm 0.8$ & $6.7 \pm 0.7$ & $7.9 \pm 0.9$ \\
$\begin{array}{c}\text { Minute ventilation } \\
\text { (mL/min/kg) }\end{array}$ & $464 \pm 50$ & $344 \pm 25$ & $341 \pm 43$ \\
$\begin{array}{c}\text { Dynamic lung com- } \\
\text { pliance }(\mathrm{mL} / \mathrm{cm}\end{array}$ & $1.8 \pm 0.1$ & $0.6 \pm 0.1$ & $0.7 \pm 0.1$ \\
$\left.\quad \mathrm{H}_{2} \mathrm{O} / \mathrm{kg}\right)$ & & & \\
\hline
\end{tabular}

$*$ Mean \pm SEM.

binding, but this test is also the least stringent for defining label bound to CLSE. Both dialysis and TCA precipitation indicate binding of $93-94 \%$ of label to large molecules or molecular aggregates in CLSE. This degree of binding was similar to that found with $\mathrm{CA}$ electrophoresis, which revealed a major band migrating at $5-10 \mathrm{~mm}$ toward the cathode that was associated with the largest fraction of ${ }^{99 \mathrm{~m}} \mathrm{Tc}$ label. This assay distinguishes protein- or lipoprotein-bound ${ }^{99 \mathrm{~m}} \mathrm{Tc}$ from other species and firmly establishes the direct labeling of CLSE constituents for distributional assessments. Labeled surfactant was also treated under very stringent conditions with organic solvent extraction (chloroform-methanol) and still almost $60 \%$ of ${ }^{99 \mathrm{~m}} \mathrm{Tc}$ remained bound directly to surfactant components.

Table 2 shows the results of physiologic measurements in the piglets immediately before lavage, and then after endogenous surfactant had been depleted by BAL. Tabulated results are for experimental animals before and after BAL, with CMV used as a common modality to this point in the experiment. As shown, BAL was associated with severe decreases in oxygenation and pulmonary mechanics in all animals. In addition, surfactant replacement therapy after lavage resulted in significant improvement in oxygenation in animals receiving either CMV or HFJV $(n=11)$. Postsurfactant results in Table 2 are combined for these piglets because there was no statistically significant difference between the two ventilation groups. The initial kinetics of distribution of labeled CLSE after tracheal instillation are shown by radioscintigraphic images in Figure 1 . The images in this figure are for the first $20 \mathrm{~s}$ of surfactant distribution after instillation in a representative piglet, and they display the extreme rapidity with which the distribution process was found to proceed in all animals. At $5 \mathrm{~s}$ after instillation (Fig. $1 A$ ), the surfactant was seen in the trachea and in the right and left mainstem bronchi. At $10-15 \mathrm{~s}$ (Fig. $1 B$ and $C$ ), surfactant was entering the right and left lungs, and the 20 -s image (Fig. $1 D$ ) shows that surfactant has reached all areas of both lungs. A small amount of free Tc was rapidly absorbed into the piglets and provided a background silhouette of the animal. This background image was eliminated for Figure 1, but did reveal that surfactant had distributed to even the peripheral areas of both lungs.

Figure 2 shows results for the ${ }^{99 \mathrm{~m}} \mathrm{Tc}$ activity in five regions of the pulmonary system over the first minute of labeled surfactant distribution after instillation. The data show measurable activity 


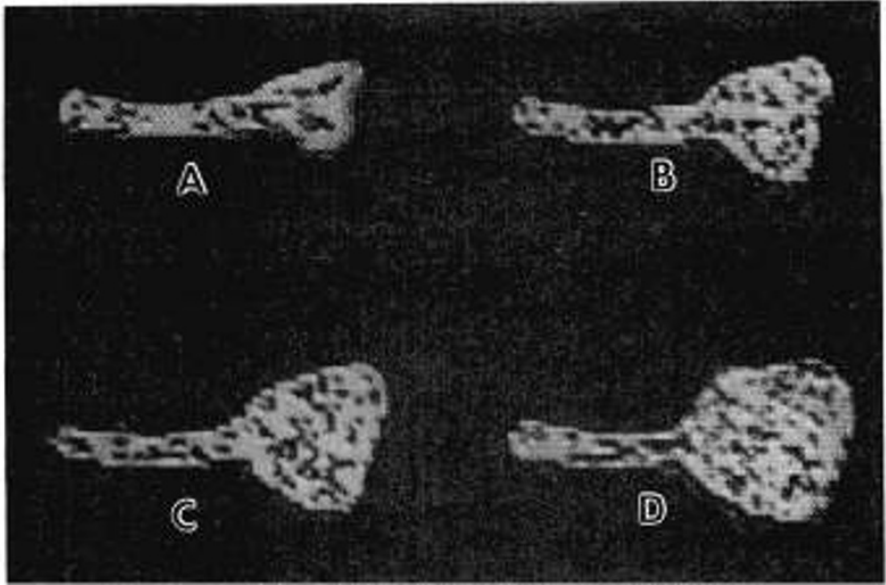

Fig. 1. Dynamic imaging of ${ }^{99 \mathrm{~m}} \mathrm{Tc}$-labeled surfactant at $5(A), 10(B)$, $15(C)$, and $20(D) \mathrm{s}$ after tracheal instillation in a representative piglet. Note complete filling of both lungs by $20 \mathrm{~s}$. Black areas are not filling defects, but colored areas of radioactivity (not demonstrable on black and white photo).

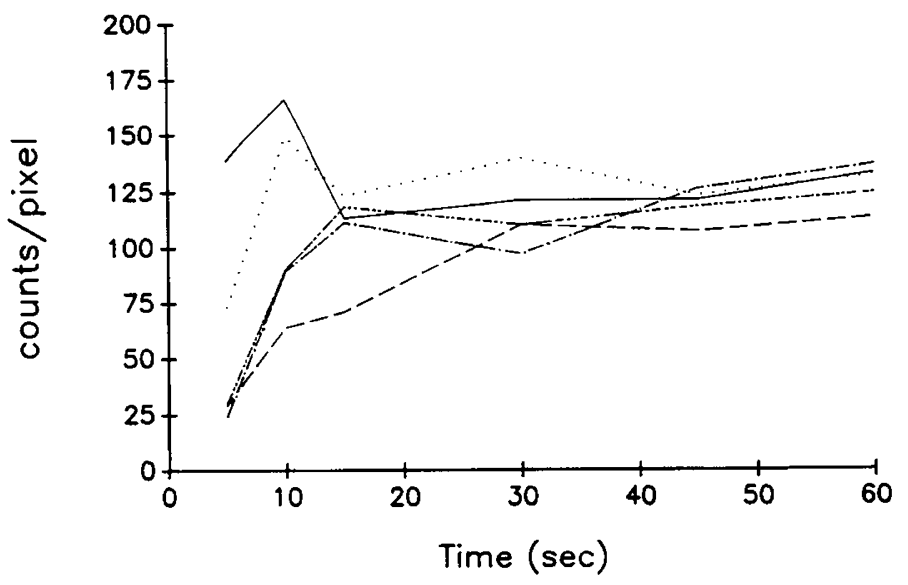

Fig. $2 .{ }^{99 \mathrm{~m}} \mathrm{Tc}$ activity in five regions of the lung over the first minute after labeled surfactant instillation. Central region, solid line; right upper lobe, dotted line; right lower lobe, dashed-dotted line; left lower lobe, long dashed line; left upper lobe, short dashed line. Results are shown for a representative piglet with no variation between CMV and HFJV animals. See text for details.

outside the central region (trachea, bronchi, and surrounding lung) even at the 5-s point, with activity reaching a plateau in all lung fields (right and left upper and lower lobes) by 20-30 s. The time scale of Figure 2 is extended over the 30-min dynamic imaging period in Figure 3, which displays the relatively symmetrical distribution of labeled CLSE into all lung areas. The ${ }^{99 \mathrm{~m}} \mathrm{Tc}$ data in Figures 2 and 3 are each for a representative animal, but results were similar in all animals studied after surfactant instillation, regardless of whether they received CMV or HFJV.

At the end of the dynamic imaging period, animals were analyzed for cumulative radiolabel over a more accurate, constant 5-min counting period to obtain a measure of relative surfactant in various regions of the lung. The results in Table 3 show that approximately $30 \%$ of the total counts were located in the central portions of the lungs at $30 \mathrm{~min}$, with the remainder distributed relatively symmetrically throughout the lungs. Again, there was no statistical difference in this count distribution between CMV and HFJV animals, which are thus combined together in Table 3. The three piglets receiving ${ }^{99 \mathrm{~m}} \mathrm{Tc}$ label in saline and $30 \mathrm{~min}$ of ventilation showed similar rapid filling of large airways in the first $10 \mathrm{~s}$, as was seen after CLSE administration (Fig. 4). However, distribution after $10 \mathrm{~s}$ was nonuniform, with multiple filling defects noted throughout areas of both lungs

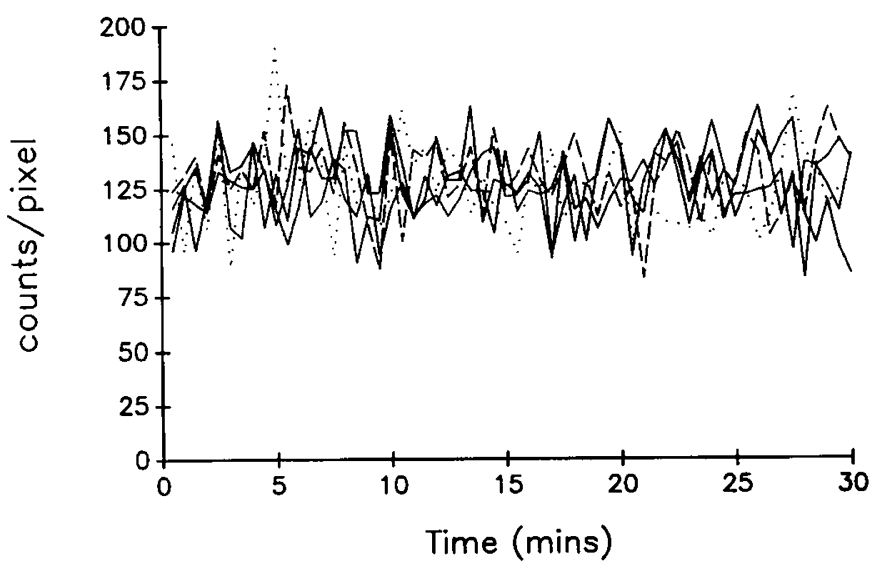

Fig. 3. ${ }^{99 \mathrm{~m}} \mathrm{Tc}$ activity in five pulmonary regions over the first $30 \mathrm{~min}$ after labeled surfactant instillation. Results show a rapid and uniform distribution to all regions of the lung. Data from a representative piglet are shown, with no significant variation found between CMV and HFJV animals.

in dynamic imaging. Statistical analyses at 30 min showed uneven distribution of radiolabel with considerable variability throughout the five regions of interest. The total number of counts was considerably lower in the saline group compared with the surfactant-treated piglets ( 37032 versus 77543 counts/pixel), presumably due to increased absorption of unbound Tc.

After counting was complete, lung microscopy was performed as shown in Figure 5. The representative results in panels $A$ and $B$ of Figure 5 show that compared with normal lung, piglets after BAL had diffuse, moderate atelectasis in all areas of the lung examined. Panel $C$ shows that surfactant replacement and 30 min of conventional ventilation resulted in significantly improved inflation patterns and much less focal atelectasis, as did the combination of surfactant and $30 \mathrm{~min}$ of HFJV in panel $D$. These results are consistent with the radiokinetic and oxygenation data suggesting that surfactant was widely distributed throughout the lungs and improved inflation patterns and gas exchange.

\section{DISCUSSION}

The results of this study indicate that tracheally instilled exogenous surfactant is rapidly distributed in a relatively symmetrical fashion in animals receiving either CMV or HFJV. Dynamic imaging, which measured radioactive counts over short periods (5) of time, demonstrated that significant amounts of surfactant were distributed to all lung fields within $20 \mathrm{~s}$ after instillation (Fig. 2). After a rapid plateau, subsequent measurements over 30 min showed continued distribution (Fig. 3) with static counting at the end of the period, indicating relatively symmetric distribution with $30 \%$ of label located centrally, $40 \%$ in the upper lobes, and $30 \%$ in the lower lobes. In contrast, at $30 \mathrm{~min}$, the saline controls showed less than $20 \%$ of the counts in the central region, almost $60 \%$ in the upper lobes, and approximately $20 \%$ of the counts in the lower lobes. Although the imaging technique used here was qualitative and involved only two dimensions, the improved oxygenation and lung morphology also observed after surfactant administration indicated that these distributional events were occurring throughout the lungs.

The rapid time scale and relatively symmetrical movement into the lungs in our study was essentially unchanged in the presence of two distinct kinds of mechanical ventilation, CMV and HFJV, which should generate respiratory flows with different microscale mixing and transport characteristics. This lack of dependence on ventilation technique, coupled with the measured kinetics of surfactant movement into the lungs, suggests that interfacial spreading phenomena are actively involved in the distribution process. Surfactants lower surface tension in propor- 
Table 3. Static counts of ${ }^{99 \mathrm{~m}}$ Tc activity in pulmonary regions after $30 \mathrm{~min}^{*}$

\begin{tabular}{|c|c|c|c|c|c|c|}
\hline & Total counts & Central & RUL & RLL & LLL & LUL \\
\hline Surfactant & 77543 & $\begin{array}{c}23062 \pm 1882 \\
(30 \%)\end{array}$ & $\begin{array}{c}16757 \pm 1052 \\
(22 \%)\end{array}$ & $\begin{array}{c}12623 \pm 2713 \\
(16 \%)\end{array}$ & $\begin{array}{c}10801 \pm 1558 \\
(14 \%)\end{array}$ & $\begin{array}{c}14300 \pm 2450 \\
(18 \%)\end{array}$ \\
\hline Saline & 37032 & $\begin{array}{c}6670 \pm 233 \\
(18 \%)\end{array}$ & $\begin{array}{c}11361 \pm 7473 \\
(31 \%)\end{array}$ & $\begin{array}{c}4269 \pm 1267 \\
(11 \%)\end{array}$ & $\begin{array}{c}5377 \pm 227 \\
(15 \%)\end{array}$ & $\begin{array}{c}9355 \pm 4144 \\
(25 \%)\end{array}$ \\
\hline
\end{tabular}

* Counts/pixel over 5-min period are shown for five lung regions: central-trachea, bronchi, and surrounding lung. RUL, right upper lobe; RLL, right lower lobe; LLL, left lower lobe; LUL, left upper lobe. Values are mean \pm SEM for surfactant treated piglets $(n=11)$ or three animals who received $\mathrm{Tc}$ mixed with saline. Values in parentheses are percentage of total counts.

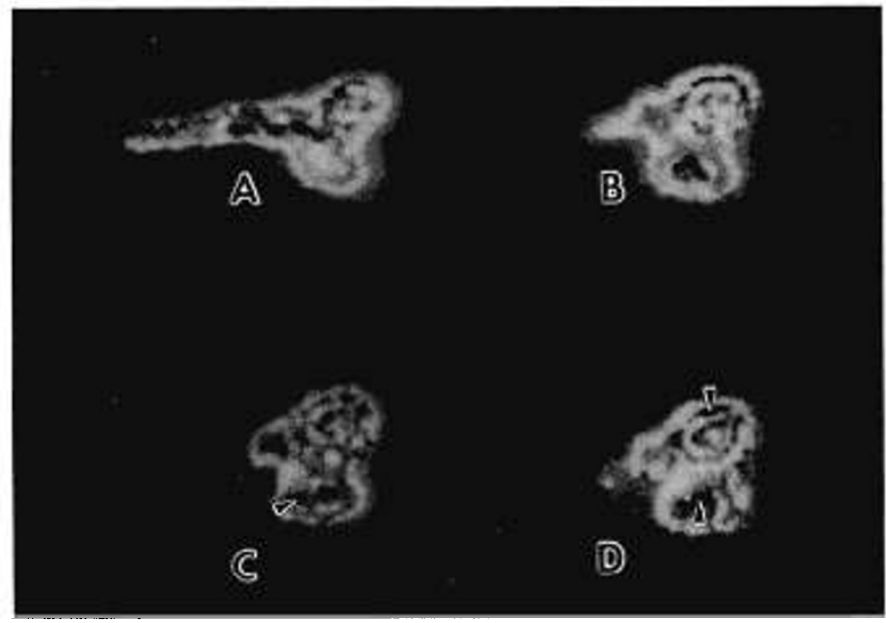

Fig. 4. Dynamic imaging of ${ }^{99 \mathrm{~m}} \mathrm{Tc}$ and saline at $5(A), 10(B), 15(C)$, and $20(D) \mathrm{s}$ after tracheal instillation in a representative piglet. Note black filling defects (arrows) in the right lung as well as the left lung.
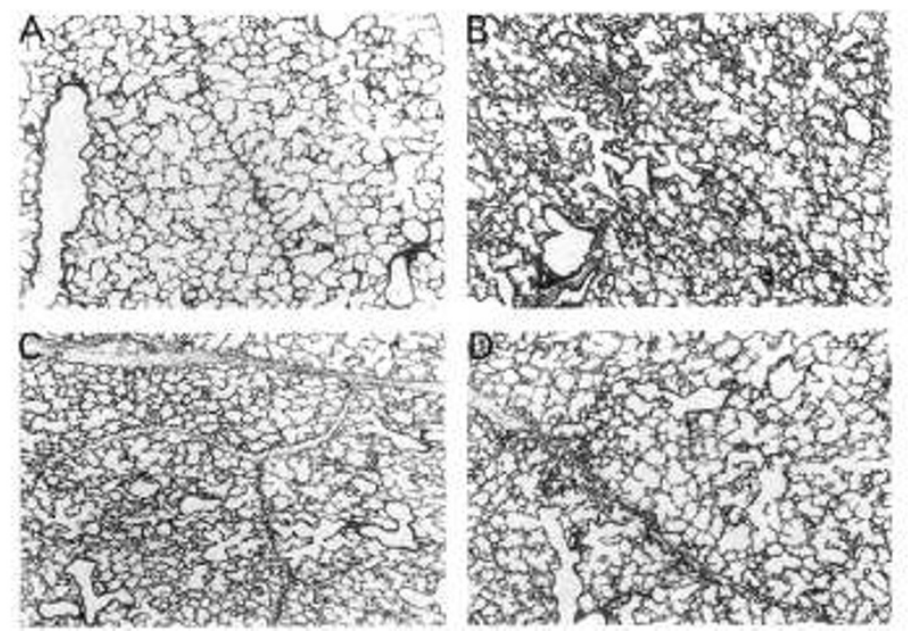

Fig. 5. Pulmonary microscopic characteristics in experimental animals. $A$, Normal inflation patterns from an unventilated control animal; $B$, moderate atelectasis after BAL; $C$, improved inflation patterns after surfactant replacement and $30 \mathrm{~min}$ of CMV; and $D$, similarly improved inflation patterns after surfactant and HFJV $(\times 100)$.

tion to their interfacial concentrations, and addition of surfactant to one region creates surface tension gradients relative to other regions. In the present experiments, such a driving force is present for the spreading of tracheally instilled surfactant, which is continually directed toward the lung periphery, and this apparently contributes to the extremely rapid initial distribution of instilled CLSE. Instillation of ${ }^{99 \mathrm{~m}} \mathrm{Tc}$ in saline alone resulted in nonuniform distribution, and again indicated that the distribution process for surfactant dispersions was not solely dependent on mechanisms of bulk flow.

Walther et al. (20) have previously studied surfactant distribution by conventional and high-frequency oscillatory ventilation using ${ }^{14} \mathrm{C}$-labeled surfactant in premature lambs with RDS.
Their results also did not indicate major differences in surfactant distribution between CMV and high-frequency ventilation, although the dynamics of distribution were not studied. Charon et al. (21) used ${ }^{99 \mathrm{~m}} \mathrm{Tc}$-labeled surfactant to evaluate exogenous surfactant distribution in newborn infants with complicated RDS. However, labeled surfactant was prepared by simple comixing ${ }^{99 \mathrm{~m}} \mathrm{Tc}$ and surfactant, and the possibility exists that the surfactant and ${ }^{99 \mathrm{~m}} \mathrm{Tc}$ dispersion could separate during distribution. The stannous reduction labeling technique described here permits binding of ${ }^{99 \mathrm{~m}} \mathrm{Tc}$ directly to surfactant components and represents a more accurate labeling method.

It is of interest that despite significant improvements in gas exchange and atelectasis, dynamic pulmonary mechanics did not improve back toward prelavage levels after surfactant administration when mechanical ventilator breaths were specifically evaluated. This is consistent with previous reports on dynamic compliance in infants $(4,21)$, and most likely represents acute lung overdistention in some regions in the presence of assisted ventilation. However, the improved oxygenation found after surfactant administration implies that underlying mechanical changes do occur, and a number of previous studies have shown explicitly that CLSE instillation improves static pulmonary mechanics in lavaged, surfactant-deficient animals lungs $(11,22$, 23).

One potential significance of the distributional results here concerns clinical exogenous surfactant replacement therapy applications. For example, exogenous surfactant is typically administered to infants with RDS in four divided intratracheal aliquots over a 5 -min period, with the infant being moved to a new position (i.e. supine, right lateral decubitus, left lateral decubitus, supine) with each aliquot to promote optimal distribution (4-8, 24). The current results suggest that exogenous CLSE distributes rapidly into the lungs even after intratracheal administration as a single bolus without positional adjustments. It is important to note that not all exogenous surfactants may distribute as rapidly as CLSE. It should also be stressed that the experimental methodology used here was designed to follow the kinetics of surfactant movement and not to provide precise information on ultimate deposition as a function of specific position in the lungs. The technique used here cannot provide microscopic resolution and some maldistribution of surfactant may have occurred both within and outside of the particular regions of interest. For complete information, the techniques here need to be coupled with more detailed methodologies involving quantitative analysis of labeled surfactant (i.e. tritium or ${ }^{14} \mathrm{C}$ labeling) in multiple lung sections.

Jobe et al. (25) studied exogenous surfactant distribution in preterm lambs using ${ }^{14} \mathrm{C}$ - and tritium-labeled surfactant. They found relatively homogeneous distribution of material (counting $1-\mathrm{cm}^{3}$ pieces of lung) when administered before the first breath, compared with inhomogeneous distribution found after mechanical ventilation had started. Lewis et al. (26) studied distribution of aerosolized surfactant versus tracheal instilled surfactant using a similar technique. There were no striking differences in distribution between the experimental groups (although comparable physiologic changes occurred in the aerosol group at a lower surfactant dose). They concluded that even these techniques for assessing surfactant distribution are of low resolution relative to actual surfactant distribution at the alveolar level. In that study, 
relatively more surfactant was detected in the right upper lobe, which was similar to what we showed using a completely different distributional assessment technique. Using the same ${ }^{14} \mathrm{C}$-labeling technique, Gilliard et al. (27) demonstrated a direct correlation between surfactant volume and homogeneity of surfactant distribution. Our study was specifically designed to look at dynamic distributional events, and a combination of techniques will be necessary to provide more optimal information on ultimate surfactant distribution.

Future work of this kind is needed particularly for adult RDS applications, where the distribution of tracheally instilled exogenous surfactant may be more uneven than it is when given early in the course of RDS $(22,28)$. In general, a uniform and symmetrical distribution profile with substantial alveolar deposition is needed for optimal surfactant therapy, and also for the use of surfactants to simultaneously deliver other respiratory drugs, such as antioxidant enzymes, to the lung.

In conclusion, a technique has been described in which exogenous surfactant has been labeled directly with ${ }^{99 \mathrm{~m}} \mathrm{Tc}$ and followed with radioscintigraphy to evaluate the dynamics of exogenous surfactant distribution after tracheal instillation. The results show that instilled CSLE distributes rapidly into the lungs with a symmetrical pattern of movement, and is associated with significant improvements in oxygenation and lung inflation patterns. The extremely short time scale of initial movement and its substantial independence of ventilation modality imply that interfacial spreading effects are prominent in this process. This may perhaps help to explain the effectiveness that has generally been observed for surfactant replacement therapy using tracheal instillation to aerated lungs.

Acknowledgments. The authors thank Dr. Neil Johnson, Dr. Donald Shapiro, Dr. Robert Notter, Susan Richter, and Jennifer Becker for their assistance in various aspects of this work.

\section{REFERENCES}

1. Farrell PM (ed) 1982 Lung Development: Biological and Clinical Perspectives, Vols I and II. Academic Press, New York

2. Notter RH 1989 Physical chemistry and physiological activity of pulmonary surfactants. In: Shapiro DL, Notter RH (eds) Surfactant Replacement Therapy. AR Liss, Inc, New York, pp 19-70

3. Notter RH, Shapiro DL 1987 Lung surfactant for replacement therapy: biochemical, biophysical, and clinical aspects. Clin Perinatol 14:433-479

4. Davis JM, Veness-Meehan K, Notter RH, Bhutani VK, Kendig JW, Shapiro DL 1988 Changes in pulmonary mechanics after the administration of surfactant to infants with respiratory distress syndrome. $N$ Engl $J$ Med 319:476-479

5. Egan EA, Notter RH, Kwong MS, Shapiro DL 1983 Natural and artificial lung surfactant replacement therapy in premature lambs. J Appl Physiol 55: 875-883

6. Hallman M, Merritt TA, Jarvenpaa AL, Boynton B, Mannino F, Gluck L, Moore T, Edwards D 1985 Exogenous human surfactant for treatment of severe respiratory distress syndrome: a randomized prospective clinical trial. J Pediatr 106:963-969
7. Kwong MS, Egan EA, Notter RH, Shapiro DL 1985 Double-blind clinical trial of calf lung surfactant extract for the prevention of hyaline membrane disease in extremely premature infants. Pediatrics 76:585-592

8. Shapiro DL, Notter RH, Morin III FC, Deluga KS, Golub LM, Sinkin RA, Weiss KI, Cox C 1985 Double-blind, randomized trial of a calf lung surfactant extract administered at birth to very premature infants for prevention of respiratory distress syndrome. Pediatrics 76:593-599

9. Bancalari E, Goldberg RN 1987 High frequency ventilation in the neonate. Clin Perinatol 14:581-598

10. Froese $A B, B r y a n A C 1987$ High frequency ventilation. Am Rev Respir Dis 135:1363-1374

11. Notter RH, Egan EA, Kwong MS, Holm BA, Shapiro DL 1985 Lung surfactant replacement in premature lambs with extracted lipids from bovine lung lavage: effects of dose, dispersion technique, and gestational age. Pediatr Res 19:569-577

12. Kowalsky RJ, Creekmore JR 1982 Technical artifacts in chromatographic analysis of Tc-99m radiopharmaceuticals. J Nucl Med Technol 10:15-17

13. Bligh EG, Dyer WJ 1959 A rapid method of total lipid extraction and purification. Can J Biochem Physiol 37:911-917

14. Bhutani VK, Abbasi S, Sivieri EM 1988 Continuous skeletal muscle paralysis: effects on neonatal pulmonary mechanics. Pediatrics 81:419-422

15. Bhutani VK, Sivieri EM, Abbasi S, Shaffer TH 1988 Evaluation of neonatal pulmonary mechanics and energetics: a two factor least mean square analysis. Pediatr Pulmonol 4:150-158

16. Lachmann B, Robertson B, Vogel J 1980 In vivo lung lavage as an experimental model of the respiratory distress syndrome. Acta Anaesthesiol Scand 24: 231-236

17. Chen PS, Toribara TY, Huber W 1956 Microdetermination of phosphorus. Anal Chem 28:1756-1758

18. Coalson JJ, Kuehl TJ, Escobedo MB, Hilliard JL, Smith F, Meredith K, Null DM, Walsh W, Johnson D, Robotham JL 1982 A baboon model for bronchopulmonary dysplasia. II. Pathologic features. Exp Mol Pathol 37:335-350

19. Walther FJ, Kuipers IM, Gidding CEM, Willebrand D, Buchholtz RTF, Bevers EM 1987 A comparison of high-frequency oscillation superimposed onto backup mechanical ventilation and conventional mechanical ventilation on the distribution of exogenous surfactant in premature lambs. Pediatr Res 22:725-729

20. Charon A, Taeusch HW, Fitzgibbon C, Smith GB, Treves ST, Phelps DS 1989 Factors associated with surfactant treatment response in infants with severe respiratory distress syndrome. Pediatrics 83:348-354

21. Heldt GP, Pesonen E, Merritt TA, Elias W, Sahn DJ 1989 Closure of the ductus arteriosus and mechanics of breathing in preterm infants after surfactant replacement therapy. Pediatr Res 25:305-310

22. Bermel MS, McBride JT, Notter RH 1984 Lavaged excised rat lungs as a model of surfactant deficiency. Lung 162:99-113

23. Berry D, Ikegami M, Jobe A 1986 Respiratory distress and surfactant inhibition following vagotomy in rabbits. J Appl Physiol 61:1741-1748

24. Merritt TA, Hallman M, Bloom BT, Berry C, Benirschke K, Sahn D, Key T, Edwards D, Jarvenpaa AL, Pohjavuori M, Kankaanpaa K, Kunnas M, Paatero H, Rapola J, Jaaskelainen J 1986 Prophylactic treatment of very premature infants with human surfactant. N Engl J Med 315:785-790

25. Jobe A, Ikegami M, Jacobs H, Jones S 1984 Surfactant and pulmonary blood flow distributions following treatment of premature lambs with natural surfactant. J Clin Invest 73:848-856

26. Lewis JF, Ikegami M, Jobe AH, Tabor B 1991 Aerosolized surfactant treatment of preterm lambs. J Appl Physiol 70:869-876

27. Gilliard N, Richman PM, Merritt TA, Spragg RG 1990 Effect of volume and dose on the pulmonary distribution of exogenous surfactant administered to normal rabbits or to rabbits with oleic acid lung injury. Am Rev Respir Dis 141:743-747

28. Matalon S, Holm BA, Notter RH 1987 Mitigation of pulmonary hyperoxic injury by administration of exogenous surfactant. J Appl Physiol 62: $756-761$ 